\title{
Comunicación y almacenamiento de datos de un compensador vectorial
}

\section{Communication and data storage of a vector compensator}

VILLALVAZO-LAUREANO, Efrain $\dagger^{*}$, GUERRERO-DENIZ, Richard Jesus y JIMÉNEZBETANCOURT, Ramón Octavio

\section{Universidad de Colima}

ID $1^{\text {er }}$ Autor: Efrain, Villalvazo-Laureano / ORC ID: 0000-0002-5939-7503, Researcher ID Thomson: H-9391-2018, CVU CONACYT ID: 54012

ID $1^{\text {er }}$ Coautor: Richard Jesus, Guerrero-Deniz, / ORC ID: 0000-0002-4924-2620, arXiv Author ID: 2689469, CVU CONACYT-ID: 990838

ID $2^{\text {do }}$ Coautor: Ramón Octavio, Jiménez-Betancourt/ ORC ID: 0000-0002-0171-7279, Researcher ID Thomson: C-18452018, CVU CONACYT ID: 88193

DOI: $10.35429 /$ JOCT.2019.10.3.1.6

Recibido Abril 15, 2019; Aceptado Junio 30, 2019

\section{Resumen}

En el presente artículo se presenta la comunicación y utilización de una base de datos (BD) para un Compensador Vectorial. Utilizando, la comunicación serial como medio entre el compensador y la BD. Se emplea una BD en MySQL puesto que la BD esta montada en un servidor web, para posteriormente obtener la visualización de datos en una página web. Anteriormente MySQL ya ha sido utilizado para el almacenamiento de datos para dispositivos eléctricos. Para una mayor facilidad de manejo de la BD se maneja como gestor de base de datos; "XAMPP".

Compensador vectorial, Comunicación serial, Scripts, MySQL

\begin{abstract}
This paper presents the communication and using a (DB) Database to Vectorial Compensator. Using the serial communication as means between the compensator and the DB. Employing a datab in MYSQL since the DB is in a web server, later get the visual data in a web site. MySQL was used to the electric's device data storage. To greaster ease of handling of DB, employing as DataBase Management System; "XAMPP”.
\end{abstract}

Vectorial Compensator, Serial Comunicaction, Scripts, MySQL

Citación: VILLALVAZO-LAUREANO, Efrain, GUERRERO-DENIZ, Richard Jesus y JEMÉNEZ-BETANCOURT, Ramón Octavio. Comunicación y almacenamiento de datos de un compensador vectorial. Revista de Tecnologías Computacionales. 2019. 3-10:1-6.

$\dagger$ Investigador contribuyendo como primer Autor.

*Correspondencia al Autor correo electrónico:(villalvazo@ucol.mx) 


\section{Introducción}

Un SEP (Sistema Eléctrico de Potencia) se encuentra computesto por tres subsistemas: Generación de energía, transmisión y centros de carga. (Exposito 2002). Acontinuación en la Figura 1 se muestra el diagrama de los tres subsistemas que componen el SEP.

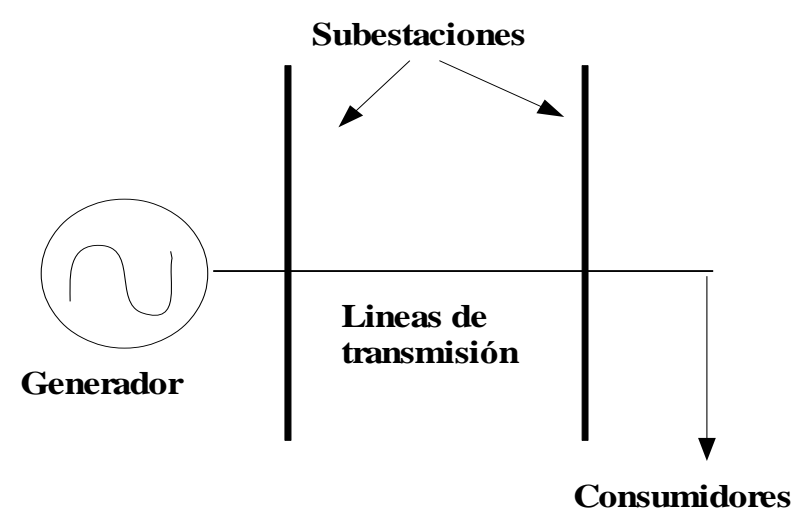

Figura 1 Sistema Electrico de Potencia

El SEP tiende a elevar los nieveles de transmisión a sus límites, es aquí el uso de condensadores en serie para su compensación. Recientemente la demanda de los SEP se ha incrementado. En la decada de los sesenta, los PSS (Sistema Estabilizador de Energía) se utilizaban para erradicar las oscilaciones por medio de provisiones de señales de control auxiliares para la exitación de los generadores. El rendimiento de los PSS esta limitado por los grandes sistemas de CA, esto afecta las variaciones de carga, cambios en los perfiles de voltaje, etc.

Los equipos FACTS (Sistemas de Transmisión de Corrriente Alterna Flexibles) son los sistemas más revelantes por su rápida respuesta, estos equipos, pueden mejorar la estabilidad en los sistemas de energía. Permitiendo un uso más eficiente en las redes de transmisión. Existen varios equipos FACTS, los más destacados son SVCs (Compensador Estático de Vars), TCSCs (Compensador Serie Controldado por Tiristor), STATCOMs (Compensador Estaático Síncrono), SSSCs (Compensador Seríe Estático Síncrono, y UPFCs (Compensador de Flujo de Potencia), unos con mayor uso que otros. Estos equipos estan hechos a base de tiristor o convertidores basados en VSC (Fuente Convertidora de Voltaje), (Ramírez, 2007). El aumento de las causas de potencia reactiva y aparente, disminuyen el factor de potencia.
Por lo tanto, la reducción de las capacidades de producción, transmisión y distribución de la línea es el resultado de los efectos del factor de potencia bajo (Ramazan Bayindir, 2011).

Los problemas técnicos que se han identificado debido al bajo factor de potencia en los sistemas de distribución de la energía eléctrica son:

- Mayor consumo de corriente.

- Aumento de las pérdidas en conductores.

- Sobrecarga de transformadores, generadores y líneas de distribución.

- $\quad$ Incremento de las caídas de voltaje.

Estos problemas conducen a que se generen los siguientes factores:

- Incremento de la facturación eléctrica por mayor consumo de corriente.

- Penalización de hasta un $120 \%$ del costo de la facturación.

Para mejorar la calidad de la energía del sistema de distribución, se aplican dos tipos de topologías de corrección del factor de potencia, la corrección del factor de potencia con dispositivos pasivos y la corrección del factor de potencia con dispositivos activos. La primera tiene una respuesta pobre dinámica y carece de regulación de voltaje. Por lo tanto, la segunda se ha utilizado para el desarrollo de más prototipos (Sujata Powniker, 2016).

Es muy importante trabajar en el diseño y aplicación de dispositivos para la corrección del factor de potencia; debido a los beneficios que se obtienen al aplicar sistemas y equipos para ayudar o corregir el problema del bajo factor de potencia; los cuales son:

- Disminución de las pérdidas en conductores.

- Reducción de las caídas de tensión.

- Aumento de la disponibilidad de potencia de transformadores, líneas y generadores.

- Incremento de la vida útil de las instalaciones.

Además de varios beneficios económicos que se presentan a continuación:

Reducción de los costos por facturación eléctrica. 
- Eliminación del cargo por bajo factor de potencia.

Bonificación de hasta un $2.5 \%$ de la facturación cuando se tenga factor de potencia mayor a 0.9 .

El promedio de las mediciones del factor de potencia en un periodo de 24 horas está usualmente por debajo del 0.9, que toma una enorme cantidad de energía adicional sancionada por las compañías de energía eléctrica (Ke Wang, 2017). Por lo que es necesario implementar dispositivos flexibles que disminuyan este problema; además de lo económico, el factor de potencia bajo incrementa los niveles de contaminación al medio ambiente; debido a la pérdida de energía eléctrica que no se transforma en trabajo.

Además de tener un buen control del factor de potencias, es conveniente ligar la información de todas las variables involcradas a una BD para su monitoreo y análisis; ya sea, si el consumo es dómestico o industrial.

Por otra parte, sabiendo que en México las tarifas del consumo de energía eléctrica son variables, según la cantidad de potencia tomada de la red eléctrica y de la temperatura promedio anual; es muy importante tener un monitoreo constante del cosnumo de energía y del fator de potencia para que el usuario lleve un buen control de su consumo e identifique los puntos críticos para su control en tiempo y forma. La tarifias se muestran en la tabla 1 .

\begin{tabular}{|l|l|l|l|l|l|l|l|}
\hline $\begin{array}{l}\text { Tarifa } \\
\text { Miles } \\
\text { pesos }\end{array}$ & $\mathbf{1}$ & $\mathbf{1 A}$ & $\mathbf{1 B}$ & $\mathbf{1 C}$ & $\mathbf{1 D}$ & $\mathbf{1 E}$ & $\mathbf{1 F}$ \\
\hline
\end{tabular}

Tabla 1 Tarifas de consumo (CFE, 2019)

Con la información mostrada en la tabla se visualiza claramente la importancia de cuidar que el cosnumo no pase lo indicado por la tarifa correspondiente a la zona en que se encuentre el consumidor; además que los consumos menores al marcado por las tarifas de la Comisión Federal de Electricidad (CFE); están subcidiados por el gobierno, en el caso de México y al sobrepasar los límites marcados el precio se incrementa considerablemente. Debido a esta situación es importante contar con un monitoreo con una BD para que el usario pueda llevar un mejor control de sus consumos, a la vez para aprovechar mejor los beneficios por bajo consumo y por tener un buen control del factor de potencia.

\section{Comunicación Serial}

La transmisión serial consiste en un protocolo muy común, que establece la comunicación entre dispositivos que se incluye de forma estándar en las computadoras. En estos puertos los bytes de información son enviados y recibidos a un bit a la vez. Este modo de comunicación es más lento que el paralelo, pero es una forma de comunicación sencilla y puede alcanzar mayor distancia.

Los datos obtenidos de forma serial son captados por la computadora, lo siguiente sería el diseño y creación de la BD. Para esto se emplea el uso del paquete XAMPP, el cual proporciona un servidor web de BD MySQL en el que se puede almacenar y administrar la BD (Helma Spona, 2010); para la elaboración de la BD y con ello las tablas de los campos pertenecientes a los valores reales captados, se usó el lenguaje SQL.

Primero, se establece una conexión serial para recibir los valores, en este caso de un Arduino Leonardo, que técnicamente adquiere valores de voltaje obtenidos de un potenciómetro que el mismo Arduino alimenta y su salida es recibida por la entrada A0 (una de las entradas analógicas de Arduino). Es importante aclarar que esto es un prototipo muy aproximado de los valores reales con los que se trabaja en el proyecto, en el cual se manejan datos de voltaje, corriente y el factor de potencia; como se puede observar en la Figura 2:

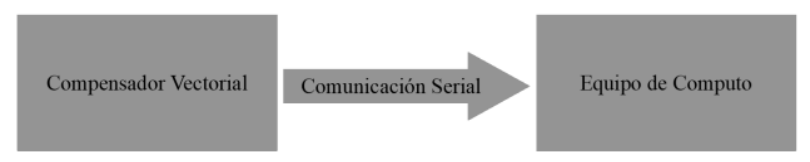

Figura 2 Diagrama de flujo sobre la comunicación entre ambos dispositivos.

Esta comunicación se realizó por medio de Python con la librería "pyserial". Con está librería por medio de una variable se obtienen datos del puerto, que en este caso es "COM3", puerto que maneja Arduino. Con ayuda de la función "serial.Serial ("variable del puerto","número de baudios")". Aquí se colocan los datos con los que se esta realizando la comunicación serial, en este caso es el puerto del Arduino y los baudios. 


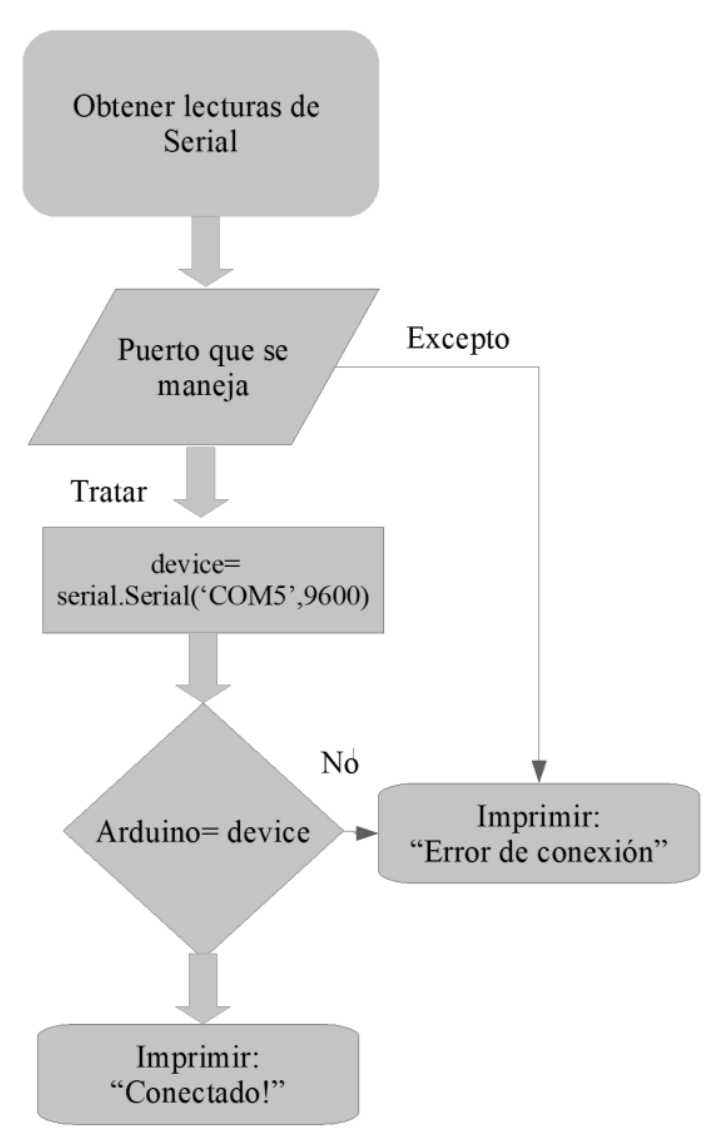

Figura 3 Variable donde almacena el puerto del Arduino, para establecer la conexión

Con siguiente comando, se imprimen todos los valores recibidos por Arduino; print("variable_puerto".readline())

\section{Datos enviados a la base de datos}

Una vez, hecho lo anterior, por medio de Python se obtienen los datos del puerto serial, se continua por medio de Python a insertar estos valores a una base de datos. Para ello se utiliza la librería "mysql.connector" y con ayuda de otra librería "date" de Python para ir registrando la fecha de registro de los datos.

Se comienza creando una base de datos, contemplando los datos del puerto serial; para este caso, los campos para el almacenaje de la fecha. Por medio de Xampp, se crea la BD con los siguientes campos; id, hora, voltaje y tiempo. En el campo de "hora" almacena la fecha obtenida por la función de Python y en "tiempo" es la fecha registrada por el servidor. Recordar que esta base de datos es sólo una prueba, simplemente como comprobación del almacenamiento de los valores obtenidos por serial.

\begin{tabular}{|l|l|l|l|}
\hline \multicolumn{1}{|c|}{ Id Hora } & \multicolumn{1}{c|}{ Voltaje } & \multicolumn{1}{c|}{ Tiempo } \\
\hline 1 & $28-11-2018$ & 0.59 & $\begin{array}{l}2018-11-28 \\
20: 55: 14\end{array}$ \\
& $20: 55: 13$ & & $\begin{array}{l}2018-11-28 \\
20: 55: 19\end{array}$ \\
\hline 2 & $28-11-2018$ & 0.79 & $2018-11-29$ \\
& $20: 55: 14$ & & $11: 38: 42$ \\
\hline 3 & $29-11-2018$ & 0.1 & $2018-11-29$ \\
& $13: 38: 38$ & & $11: 38: 47$ \\
\hline 4 & $29-11-2018$ & 0.1 & $2018-11-29$ \\
& $13: 38: 42$ & & $11: 38: 52$ \\
\hline 5 & $29-11-2018$ & 0.1 & $2018-11-29$ \\
& $13: 38: 47$ & & $11: 38: 57$ \\
\hline 6 & $29-11-2018$ & 0.1 & $2018-11-29$ \\
& $13: 38: 52$ & & $11: 39: 02$ \\
\hline 7 & $29-11-2018$ & 0 & $2018-11-29$ \\
& $13: 38: 57$ & & $11: 39: 07$ \\
\hline 8 & $29-11-2018$ & 1.05 & $2018-11-29$ \\
& $13: 39: 02$ & & $11: 39: 12$ \\
\hline 9 & $29-11-2018$ & 1.04 & \\
& $13: 39: 07$ & & \\
\hline
\end{tabular}

Tabla 2 Estructura de la BD con registro de voltaje junto con la fecha obtenida una por Python(hora) y otra por el servidor (tiempo)

Una vez que nuestra BD esta estructurada, se continua con la conexión a ella, cómo se muestra acontinuación:

mydb $=$ mysql.connector.connect(host="localhost",user ="root",passwd="',database="prueba")

Una vez establecida la conexión se hace uso de la función "crusor()" para ejecutar los comandos de inserción; aclarando que se utiliza una sesión iniciada de la DB. Para el cálculo de tiempo se utiliza la siguiente función "datetime.now().strftime("\%d-\%m-\%Y

$\% \mathrm{H}: \% \mathrm{M}: \% \mathrm{~S} ")$ ", en donde se obtiene; día, mes, año, hora, minutos y segundos, almacenados en una variable (datetime es el nombre que usa de la librería "date").

Con los datos leídos del serial y conjunto la fecha se procede a insertarlo en la BD. A continuación, se muestra un diagrama de flujo qué integra toda la función: 


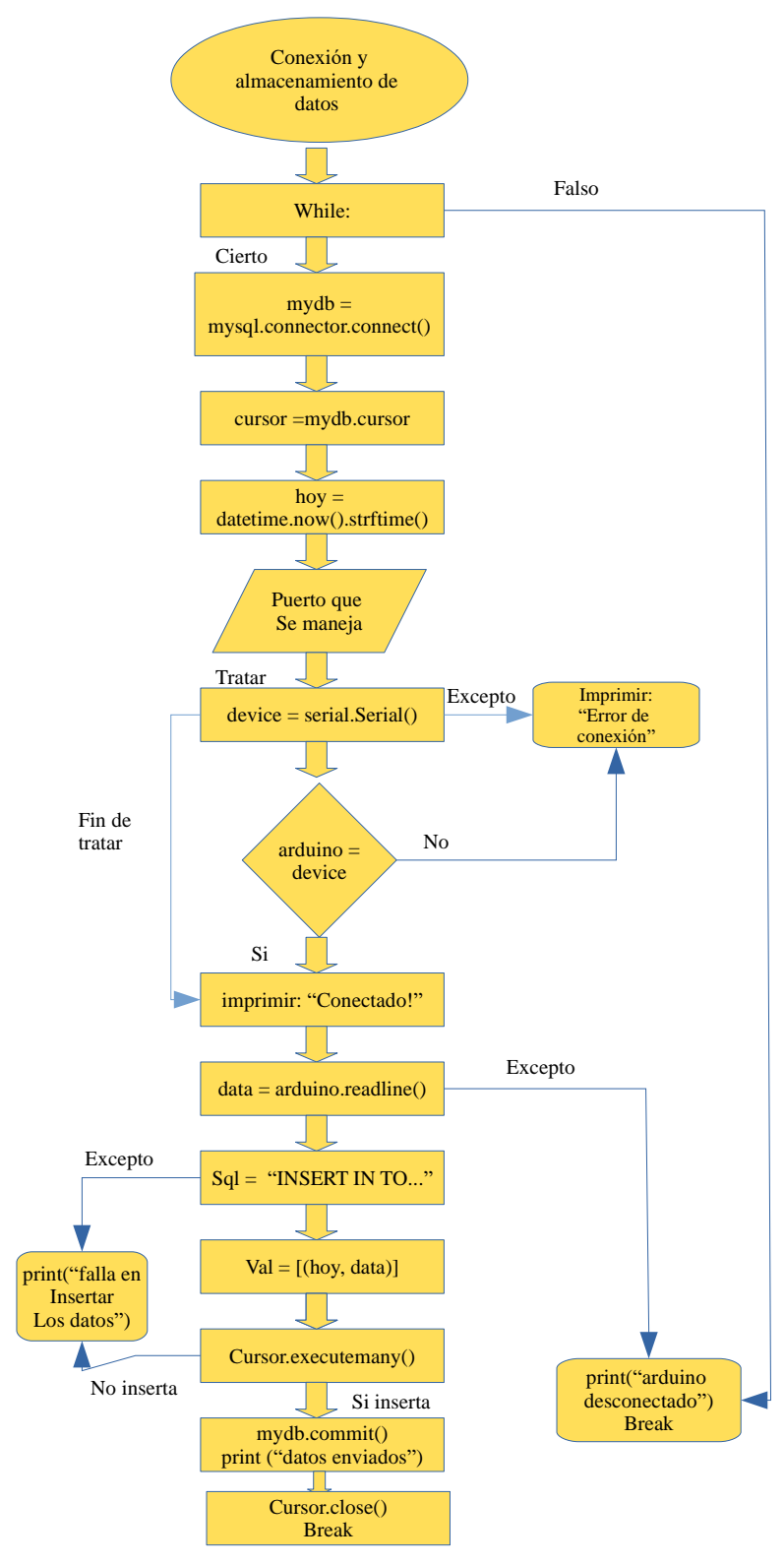

Figura 4 Diagrama de flujo que explica toda la función

\section{Presentación de resultados}

Los resultados obtenidos son visibles en la Figura 3. Estos datos cuentan con una identificación unica para diferenciarlos de los demás y cómo se observa en la BD, cuentan con dos tipos de fechas de registros, con la diferencia en que, dentro del campo "tiempo" se encuentra la fecha registrada por el servidor y en el campo de "Hora" se encuentra la fecha registrada por el programa.

Cómo se mencionó anteriormente, estos datos recopilados son parte de un prototipo muy próximo de los datos reales que el compensador esté manejando, sin embargo ya se establece una comunicación con un dispositivo que es similar al compensador, que también, almacena en una BD con los mismos tipos de valores que el compensador maneja.

\section{Agradecimientos}

A la Universidad de Colima y PROMEP por todo el apoyo brindado para la elaboración de este trabajo de investigación entre esudinates y académicos.

\section{Conclusiones}

- La comunicación serial en este caso es el más recomendable para el compensador por la facilidad que se le presenta establecer una conexión con un equipo de cómputo.

- El tiempo de ejecución del código para lectura e insersíon de datos depende la programación que cuente el compensador vectorial.

- Es una BD que se puede modificar fácilmente por ser de arquitectura abierta.

- Facilitará el manejo y análisis de datos por parte de los usuarios.

- Contribuirá al deseño de dispositivos más eficientes para el ahorro de energía con el aumento del factor de potencia.

\section{Referencias}

Bolton, W. (2012). Mecatrónica. México: Alfaomega.

Boylestad, R. L. (2009). Electrónica: Teoría de circuitos y dispositivos electrónicos. México: Pearson.

Caballero, H. (2006). Simulación y electrónica analógica, prácticas y problemas. México: RAMA.

CFE. (16 de Febrero de 2019). Comisión Federal de Electricidad. Obtenido de https://app.cfe.mx/Aplicaciones/CCFE/Tarifas/ TarifasCRECasa/Tarifas/TarifaDAC.aspx

Coughlin, R. F. (2010). Circuitos integrados lineales $y$ amplificadores operacionales. México: Prentice-Hall.

Guerra, J. P. (2012). Electrónica analógica para ingenieros. México: McGrawHill.

Ke Wang, H. H. (2017). Study on Power Factor Behavior in High-Speed Railways. IEEE Transactions on Transportation Electrification, De la 1 a la 11. 
Malvino, A. P. (2007). Principios de electr\{onica. México: McGrawHill.

Marino, J. J. (2012). Problemas resueltos de electrónica analógica. México: ECU.

Ramazan Bayindir, E. K. (2011). An Intelligent Power Factor Correction Approach Based on Linear Regression. International Conference on Machine Learning and Applications and Workshops, 313-315.

Ramírez, J. M. (2007). Stead state and transient stability whit an AC/AC PWM series compensator . IEEE PES Summer meeting, 8.

Rico, R. P. (2007). Electrónica analógica integrada. México: Marcombo.

Sujata Powniker, S. S. (2016). Development of Active Power Factor Correction. IEEE International WIE Conference on Electrical and Computer Engineering (WIECON-ECE), 212216. 